\title{
No news is bad news
}

\author{
Curtis F. Lavoie, MD
}

See related articles on pages 523 and 545

Version française À la PAge 568

$\mathbf{P}$ ractising emergency medicine is a bit like flying a plane at low altitude ... through the Rocky Mountains ... blindfolded. You can try to avoid the danger, but the outcomes of your decisions are unknown. Until, that is, you crash into Mount Robson.

On a slow day one September, I happened upon a group of nurses and physicians where I work discussing a recent "scary case." A 9-year-old boy with a headache had been seen, treated with metoclopramide and sent home. Six hours later he returned with meningitis, required intubation and was admitted to the intensive care unit. Everyone was shaken. How could this happen? After a pause my colleague turned to me and said, "Hey Curtis, I think your name was on that kid's chart."

"Augh!!" Why didn't I listen to my mother and become a pilot?

Much has been made of the parallels between the aviation industry and the practice of emergency medicine, ${ }^{1}$ but I take exception. On one hand, pilots, like emergency physicians, undergo rigorous professional schooling and apprenticeship, and then work under high-pressure, time-constrained conditions, at odd hours, making decisions with the potential for life-and-death consequences. On the other hand, pilots get high-quality outcome feedback on every decision they make by just looking out the window; and the most important outcome, a successful landing (or not) at a planned destination, is easily observed at the end of every shift (Fig. 1).

In emergency medicine the opposite is true. Patients' conditions evolve over days to weeks, which means important diagnostic and therapeutic outcomes can occur after discharge, hand-over from our care or admission to hospital. Furthermore, we don't usually see our patients again, and there are no systems to tell us if things go right or wrong. Without any feedback on the outcomes of our decisions, I believe we are flying blindfolded.

To some extent all emergency physicians must assume that "no news is good news" in order to have confidence in their decisions. The problem with this "no news is good news" approach is that it assumes there is some systemic imperative to let us know when things go wrong. In other words, "Somebody would have said something by now." Unfortunately, this is flawed. Patients frequently cannot get in touch with us, colleagues may be reluctant to reveal bad news and few surveillance mechanisms exist in our health care system to report unexpected outcomes to practitioners. The "I have never been sued" defence is also unsound. Legal action is only taken if there is patient suffering, a perceived medical error and motivation to mend the wrong. Most outcomes never meet this threshold.

According to Croskerry, ${ }^{3}$ knowing outcomes is necessary to "calibrate" the decision-making tools of clinicians, an idea that comes from the decision sciences literature.

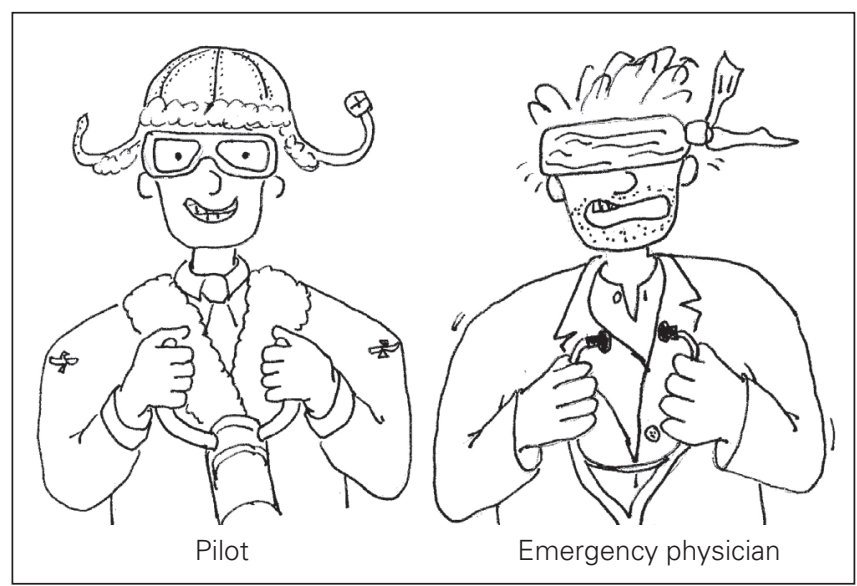

Fig. 1. Aviation versus emergency medicine.

Emergency Department, Hôpital Montfort, and Division of Emergency Medicine, Children's Hospital of Eastern Ontario, Ottawa, Ont.

All editorial matter represents the views of the authors and not necessarily those of CJEM or the Canadian Association of Emergency Physicians. 
Through calibration individuals can improve the accuracy of their probability estimates in response to outcome information. If an outcome is positive, it reinforces the decision behaviours; if it is negative, it forces reflection on the decision and potentially a modification to decision behaviours (Fig. 2). The problem is that if we don't hear otherwise, we assume the outcome is positive, which reinforces all our diagnostic and therapeutic strategies, including the unsuccessful ones. Mistakes are reinforced instead of discouraged and physicians persist with ineffective or even dangerous strategies., ${ }^{2,3}$ Better outcome feedback would point out errors and have the potential to improve diagnostic accuracy, therapeutic choices and patient safety.

In addition, calibration may have other roles, for example, in improving clinical efficiency. By knowing the outcomes for an increasing proportion of patients, physicians would gradually shift to use only the most valuable clinical clues and tests to reach the thresholds of diagnosis or treatment. Job satisfaction may also be affected: a deficit in outcome feedback limits a sense of one's impact on their community ("Am I making a difference? Who knows?!") and a sense of one's own selfcalibration or expertise ("Am I good at what I do? Who knows?!"). The lack of quality outcome feedback may also indirectly lead us to order unnecessary tests to soothe our intellectual needs or self-confidence. More reliable feedback could improve both.

Intuitively, focused attention to the outcomes of decisions is important to learning any skill. Gardeners need to see their azaleas bloom, chefs need to sample their bouillabaisse and lawyers need to read their verdicts. Yet

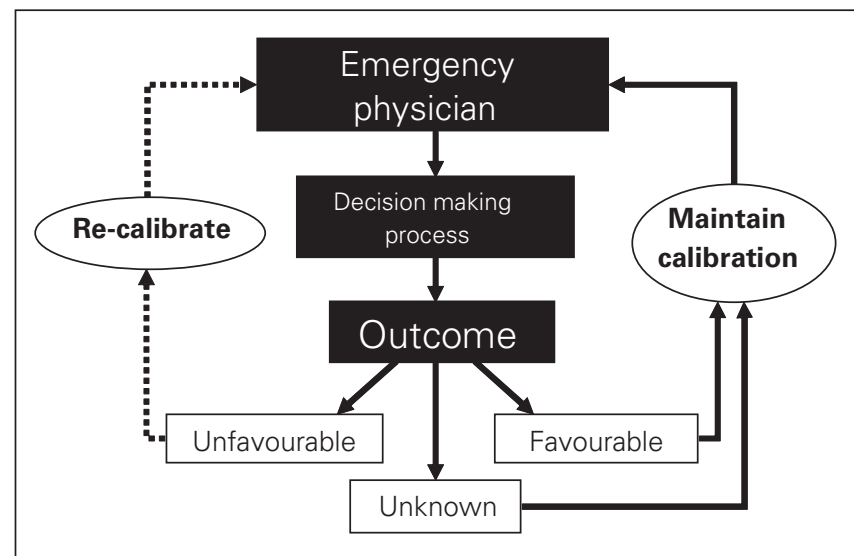

Fig. 2. Outcome feedback and calibration in emergency medicine: most outcomes are unknown and this may reinforce all decision-making strategies, even if erroneous. Reprinted with permission. ${ }^{3}$ even among medical professionals, emergency physicians stand alone. Primary care physicians follow individual patients for years, and specialists admit to hospital or follow-up with their patients to assess outcomes. Pediatricians see their patients grow, psychiatrists see their patients smile, urologists see their patients pee and rectal surgeons ... well, you get the point. Outcome feedback is so fundamental to the mastery of any profession that most do not have to think about it. In emergency medicine, however, we must.

\section{CONCLUSION}

After I heard about the child with meningitis, I went straight up to the ward. He was well; in fact, he was playing video games. I reviewed the chart, made some notes and returned to my work. What was remarkable is that feedback, in spite of the significant diagnosis, only happened by chance. The problem is this is the only way it happens.

Outcome feedback should not be left to random chance: emergency physicians cannot assume that if something bad happens to their patients, they will hear about it. Moreover "hearing about it" appears to be essential to calibrating clinical decision tools, avoiding errors and generally improving medical practice. Feedback on the outcomes of one's decisions is how most other professionals become experts. So, in the name of expertise and patient safety, we need to understand and improve outcome feedback to emergency physicians, especially when things go wrong. Only then can we take off our blindfolds and enjoy the flight.

\section{Competing interests: None declared.}

Keywords: treatment outcome, quality of health care, emergency medicine, decision support techniques, outcome feedback

\section{REFERENCES}

1. Kohn LT, Corrigan JM, Donaldson MS, editors. To err is buman: building a safer bealth care system. Washington (DC): National Academy Press; 2000.

2. Rudolph JW, Morrison JB. Sidestepping roadblocks: a feedback model of diagnostic problem solving. Am 7 Med 2008; 121:S34-7.

3. Croskerry P. The feedback sanction. Acad Emerg Med 2000; $7: 1232-8$.

Correspondence to: Dr. Curtis Lavoie, Hôpital Montfort, 713 Montreal Rd., Ottawa ON K1K 3E9; curtislavoie@gmail.ca 